\title{
Human pancreatic adenocarcinoma contains a side population resistant to gemcitabine
}

\author{
Anke Van den broeck ${ }^{1,2}$, Lies Gremeaux², Baki Topal ${ }^{1 *}$ and Hugo Vankelecom²
}

\begin{abstract}
Background: Therapy resistance remains one of the major challenges to improve the prognosis of patients with pancreatic cancer. Chemoresistant cells, which potentially also display cancer stem cell (CSC) characteristics, can be isolated using the side population (SP) technique. Our aim was to search for a SP in human pancreatic ductal adenocarcinoma (PDAC) and to examine its chemoresistance and CSC(-like) phenotype.
\end{abstract}

Methods: Human PDAC samples were expanded in immunodeficient mice and first-generation xenografts analyzed for the presence of a Hoechst dye-effluxing SP using flow cytometry (FACS). To investigate chemoresistance of the SP, mice bearing PDAC xenografts were treated with gemcitabine and SP proportion determined. In addition, the SP and the main tumour cell population (MP) were sorted by FACS for RNA extraction to profile gene expression, and for culturing under sphere-forming conditions.

Results: A SP was identified in all PDAC samples, analyzed. This SP was more resistant to gemcitabine than the other tumour cells as examined in vivo. Whole-genome expression profiling of the SP revealed upregulation of genes related to therapy resistance, apoptotic regulation and epithelial-mesenchymal transition. In addition, the SP displayed higher tumourigenic (CSC) activity than the MP as analyzed in vitro by sphere-forming capacity.

Conclusion: We identified a SP in human PDAC and uncovered a chemoresistant and CSC-associated phenotype. This SP may represent a new therapeutic target in pancreatic cancer.

Trial registration: Clinicaltrials.gov NCT00936104

Keywords: Pancreatic cancer, Chemoresistance, Side population

\section{Background}

Pancreatic cancer or ductal adenocarcinoma (PDAC) remains a highly lethal disease. Despite improvements in medical and surgical care, the overall 5-year survival still has not exceeded 5\% [1]. Resistance to chemotherapy is a major cause of treatment failure in pancreatic cancer, both in adjuvant setting after intended curative surgery as well as in advanced inoperable stages [2]. Therefore, PDAC's chemoresistant cells are highly wanted targets for new therapeutic strategies to eventually improve overall survival.

Therapeutic resistance in pancreatic cancer is caused by low permeability of the tumour micro-environment, as well as by the efficient efflux of toxic agents $[3,4]$.

\footnotetext{
* Correspondence: baki.topal@uzleuven.be

'Department of Abdominal Surgery, University Hospitals Leuven, Herestraat 49, B-3000, Leuven, Belgium

Full list of author information is available at the end of the article
}

One approach to isolate drug-effluxing cells is provided by the side population (SP) technique [5]. SP cells are identified on the basis of Hoechst-dye efflux capacity because of the presence of multidrug resistance transporters. Cells that expel the dye are visualized by dualwavelength flow cytometry (FACS) as a 'Hoechst low' tail of cells, the SP, relative to a larger bulk of 'Hoechst high' cells, the main population (MP).

Recently, a SP has been identified in cultured pancreatic cancer cell lines and was found to be chemoresistant to gemcitabine treatment as evaluated in vitro in these cultured cell lines [3,6-8]. To date, it is not known whether clinical human PDAC contains a SP and wether this SP is resistant to gemcitabine when assessed in vivo.

In multiple types of cancer the SP is enriched in cells displaying properties of cancer stem cells (CSC) $[9,10]$. By definition, CSC (also referred to as tumour-driving cells) represent the tumour's subpopulation with the

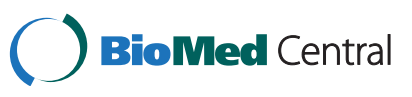


highest capacity to drive its growth, invasion and metastasis. CSC are also considered responsible for therapy resistance and disease recurrence [11], and may therefore represent interesting targets for new and more effective treatment strategies [12]. Although still subject of debate, (candidate) CSC populations are being identified in a growing number of cancer types and their functional significance has recently been strongly supported [13-15]. In pancreatic cancer, cells based on specific cell-surface markers (i.e. $\mathrm{CD} 24^{+} \mathrm{CD} 44^{+} \mathrm{ESA}^{+}$and $\mathrm{CD} 133^{+}$cells) have been reported to possess CSC characteristics $[12,16,17]$.

In the present study, we report for the first time that human PDAC contains a SP and analyzed its resistance to the current standard chemotherapeutic agent for pancreatic cancer, gemcitabine, using a PDAC xenograft in vivo model. In addition, we performed whole-genome expression analysis of PDAC SP cells, which may guide to CSC-associated characteristics and to potential therapeutic targets. Finally, we explored in vitro whether the PDAC SP is enriched in tumourigenic cells as a further characteristic of CSC.

\section{Methods}

\section{PDAC samples and xenografts}

Between 2007 and 2010, PDAC surgical resection specimens were obtained at the University Hospital Leuven (Belgium) from patients after written informed consent (see Table 1). The study was approved by the KU Leuven ethical committee prior to patient recruitment, and received the study number ML3452. Freshly resected tumours were cut into small pieces $(2 * 2 \mathrm{~mm})$ and implanted subcutaneously (s.c.) in the axilla of severe combined immunodeficiency (SCID) mice (male, 610 weeks old) to expand tumour material. Tumour growth was evaluated with a caliper on a weekly basis and volume calculated according to the formula: tumour volume $=\left(\right.$ length $\mathrm{x}$ width $\left.{ }^{2}\right) / 2$ [18]. Mice bearing tumours with a minimum volume of $150 \mathrm{~mm}^{3}$ were euthanized and tumours were dissected for further analysis. Only first-generation xenograft tumours were used in the experiments described. Hematoxylin-Eosin staining was performed on formalin-fixed sections from original and xenograft tumours.

\section{SP analysis}

Xenograft tumours $(\mathrm{n}=17)$ were dissociated into single cells using collagenase type IV $(1 \mathrm{mg} / \mathrm{ml}$ in Medium 199; Invitrogen, Grand Island, NY). Cells were incubated with Hoechst33342 (Sigma-Aldrich, Bornem, Belgium) at a final concentration of $5 \mu \mathrm{g} / \mathrm{ml}$, and the SP was identified as a side branch of 'Hoechst low' cells using dualwavelength FACS analysis (FACSVantage SE, equipped with FACS DIVA software, version 6.0; BD Biosciences, Erembodegem, Belgium; Hoechst red with 675/20 nm filter and Hoechst blue with 424/44 nm filter). Verapamil $(100 \mu M$; Sigma-Aldrich) was added to verify the SP

Table 1 Patients' characteristics with PDAC used for xenografting

\begin{tabular}{|c|c|c|c|c|c|c|c|c|c|c|c|c|c|c|c|}
\hline Xenograft no. & Sex & Age (y) & pG & pT & $\mathrm{pN}$ & $\mathrm{pM}$ & $\mathrm{pR}$ & PNI & VI & LVI & Preop RCT & Postop RCT/CT & OS (m) & DFS (m) & SP\% \\
\hline $101^{*}$ & $\mathrm{~F}$ & 45.5 & 2 & 4 & 1 & 0 & 1 & 1 & 0 & 0 & 0 & RCT & 35.4 & 18.5 & 1.5 \\
\hline $110^{*}$ & M & 77.8 & 2 & 3 & 0 & 0 & 1 & 1 & 1 & 1 & 0 & 0 & 1.1 & 1.1 & 6.0 \\
\hline $112^{*}$ & $\mathrm{~F}$ & 77.8 & 2 & 3 & 1 & 0 & 0 & 1 & 1 & 1 & 0 & $C T$ & 10.1 & 3.6 & 6.8 \\
\hline 127 & M & 52.9 & 2 & 2 & 1 & 0 & 0 & 1 & 0 & 0 & 0 & CT & 37.7 & 34.8 & 6.4 \\
\hline $128^{*}$ & M & 53.6 & 2 & 3 & 1 & 0 & 1 & 1 & 0 & 0 & 0 & $\mathrm{CT}$ & 11.2 & 10.2 & 6.8 \\
\hline $136^{*}$ & M & 78.4 & 2 & 3 & 1 & 0 & 0 & 0 & 0 & 0 & 0 & 0 & 37.8 & 24.5 & 6.1 \\
\hline $151^{\circ}$ & M & 45.9 & 2 & 3 & 1 & 0 & 0 & 1 & 1 & 1 & 0 & $C T$ & 13.5 & 10.3 & 17.6 \\
\hline 169 & $\mathrm{~F}$ & 80.5 & 2 & 3 & 1 & 0 & 0 & 0 & 1 & 1 & 0 & 0 & 10.2 & 2.4 & 4.3 \\
\hline $174^{* *, o}$ & M & 66.9 & 2 & 3 & 0 & 0 & 0 & 1 & 1 & 1 & 0 & $C T$ & 43.3 & 19.8 & 2.7 \\
\hline $178^{* *}$ & $\mathrm{~F}$ & 53.2 & 3 & 4 & 1 & 0 & 0 & 1 & 0 & 1 & 0 & $C T$ & 27.2 & 27.2 & 12.0 \\
\hline 199 & M & 52.2 & 3 & 3 & 0 & 0 & 1 & 1 & 1 & 1 & 0 & $\mathrm{CT}$ & 23.5 & 4.9 & 5.0 \\
\hline $207^{* *, o}$ & F & 67.0 & 2 & 3 & 0 & 0 & 0 & 0 & 0 & 0 & 0 & CT & 7.2 & 5.5 & 10.0 \\
\hline 218 & $\mathrm{~F}$ & 62.6 & 3 & 3 & 1 & 0 & 0 & 1 & 1 & 1 & 0 & 0 & 0.3 & 0.3 & 1.4 \\
\hline $223^{* *, o}$ & F & 57.3 & 2 & 2 & 1 & 0 & 0 & 1 & 1 & 1 & 0 & 0 & 24.5 & 7.4 & 2.0 \\
\hline $229^{* *}$ & $\mathrm{~F}$ & 73.2 & 3 & 3 & 1 & 0 & 0 & 1 & 1 & 1 & 0 & 0 & 4.5 & 4.0 & 5.4 \\
\hline $235^{* *, o}$ & F & 57.4 & 1 & 3 & 1 & 0 & 1 & 1 & 0 & 0 & 0 & $\mathrm{CT}$ & 19.6 & 7.7 & 2.1 \\
\hline $241^{* *, o}$ & F & 74.6 & 2 & 3 & 0 & 0 & 0 & 1 & 0 & 0 & 0 & 0 & 6.9 & 2.9 & 2.7 \\
\hline
\end{tabular}

* used for microarray analysis; ** used to investigate gemcitabine resistance; ${ }^{\circ}$ used for sphere-formation assay.

Abbreviations: F: female; M: male; y: year; p: pathological; G: histopathological grade; T: tumour size; N: lymph node metastasis; M: metastasis; R: resection margin; PNI: perineural invasion; VI: vascular invasion; LVI: lymphovascular invasion; RCT: radiochemotherapy; CT: chemotherapy; OS: overall survival; DFS: disease-free survival; m: month. 
phenotype, as it results in the reduction of the side branch by blocking the multidrug transporters. Propidium Iodide $(2 \mu \mathrm{g} / \mathrm{ml}$; Sigma-Aldrich) was used to exclude dead cells. For further characterization, tumour cells were immunostained for the endothelial marker CD31 and the hematopoietic marker CD45. After Hoechst incubation, fluorescein (FITC)-labeled anti-mouse or anti-human CD31 and phycoerythrin (PE)-labeled anti-mouse or antihuman CD45 antibodies (BD Biosciences), or PE-labeled anti-human CD133 (Miltenyi Biotec, Bergisch Gladbach, Germany) were added using dilutions according to the manufacturer's recommendations. Sorted SP and MP cells were established as monolayers and subjected to CytoRich Red staining (BD Biosciences).

\section{Treatment of mice bearing xenograft tumours with gemcitabine}

To investigate resistance of SP cells to gemcitabine, 7 different human PDAC samples were grown in SCID mice (see Table 1). When the tumour reached a volume of approximately $200 \mathrm{~mm}^{3}$, one group of mice received gemcitabine (Eli Lilly, Brussels, Belgium; $200 \mathrm{mg} / \mathrm{kg}$ body weight intraperitoneally, 1 injection every 3 days, 6 injections in total) and the other group (bearing the corresponding tumours) was injected with vehicle $(0.9 \%$ $\mathrm{NaCl}$; control group). Tumour diameter was measured every 3 days after the first injection. Three days after the last injection, mice were euthanized and tumours analyzed to determine the proportion of SP cells as described above. Gemcitabine was considered effective when tumour volume decreased at least 50\%.

\section{Whole-genome expression profiling}

For RNA extraction, 25000 SP and 25000 MP cells were sorted by FACS into cold lysis solution (RNeasy Micro Kit; Qiagen, Venlo, The Netherlands). RNA was extracted according to the instructions of the manufacturer. RNA quality and concentration were determined using Picochips on a BioAnalyzer 2100 (Agilent Technologies, Santa Clara, CA). Only samples with RNA Integrity Number (RIN) $\geq 8.0$ were used for gene expression profiling by microarray analysis. After Baugh amplification, Cy3 label was incorporated into the cRNA, which was then hybridized onto whole-genome human $44 \mathrm{~K}$ oligonucleotide arrays (G4112F, Agilent) [19,20].

Expression values were obtained using the Agilent feature extraction software (version 10.1.1.1) and subjected to quantile normalization. Probes lacking a detection call signal $(\mathrm{n}=1666)$ were omitted from further analysis. The log2-ratios for each SP-MP pair were compared with the Limma (Linear Models for Microarra Data) package of Bioconductor [21]. The contrast SP-MP was tested with a moderate t-statistic (implemented in Limma). The resulting p-values were corrected for multiple testing with Benjamini-Hochberg to control false discovery rate. The Database for Annotation, Visualization and Integrated Discovery (DAVID, version 6.7, http://www.david.abcc. ncifcrf.org) was used to uncover enriched function-related gene groups by gene-annotation enrichment analysis and to reveal enriched KEGG (Kyoto Encyclopedia of Genes and Genomes) pathways. Significance was tested by the EASE score, a modified Fisher's exact p-value test to adjust for multiple testing. Gene clusters with an enrichment score of $>1.5$ were retained. To further visualize gene networks, the Search Tool for the Retrieval of Interacting Genes (STRING, http://string.embl.de) was used.

Gene expression data are available from the Gene Expression Omnibus (GEO, http://www.ncbi.nlm.nih.gov/ projects/geo/) through series accession number GSE36563.

\section{Tumourigenic (CSC) activity as analyzed by in vitro sphere formation}

To investigate sphere-forming capacity, SP and MP subpopulations were sorted by FACS from PDAC xenograft tumours $(n=6$; see Table 1), and 40000 cells of each seeded in DMEM/F12 (Invitrogen), supplemented with $0.4 \%$ BSA and containing basic fibroblast growth factor (bFGF; 10 ng/ml; R\&D Systems, Minneapolis, MN), epidermal growth factor (EGF; $20 \mathrm{ng} / \mathrm{ml}$; R\&D Systems), insulin-transferrin-selenium (1:100; Invitrogen) and B27 (1:50; Invitrogen) [22]. The medium was renewed at day 3 , and spheres were counted at day 7 to determine the sphere-forming capacity.

\section{Statistical analysis}

Statistical analysis was performed using the Visual data discovery software JMP9 (SAS, Cary, NC). A Wilcoxon Test for nonparametric variables was applied to determine the statistical level of difference between values of PDAC SP and MP and to compare the results from the mouse group treated with gemcitabine and the control group. Statistical significance was determined as $\mathrm{p}<0.05$, and as $\mathrm{p}<0.001$ for the microarray analysis.

\section{Results}

\section{Presence of a SP in human PDAC xenografts}

Human PDAC samples $(\mathrm{n}=17$; Table 1$)$ were expanded by s.c. implantation, each in several SCID mice. All tumours grew in at least one mouse, regardless of the PDAC clinicopathological features or disease stage. Xenograft tumours showed histological resemblance to the original PDAC, although the stromal component was less abundant in the xenograft than in the original tumour (Figure 1A). In all tumours analyzed $(\mathrm{n}=17)$, a SP was identified, ranging from 1.4 to $17.6 \%$ of total cells (median: 5.4\%; Figure 1B-C; Table 1). Addition of verapamil during incubation with Hoechst decreased the SP proportion to $0.4 \%$ (range: $0.1-5.6 \%$ ), thus confirming 

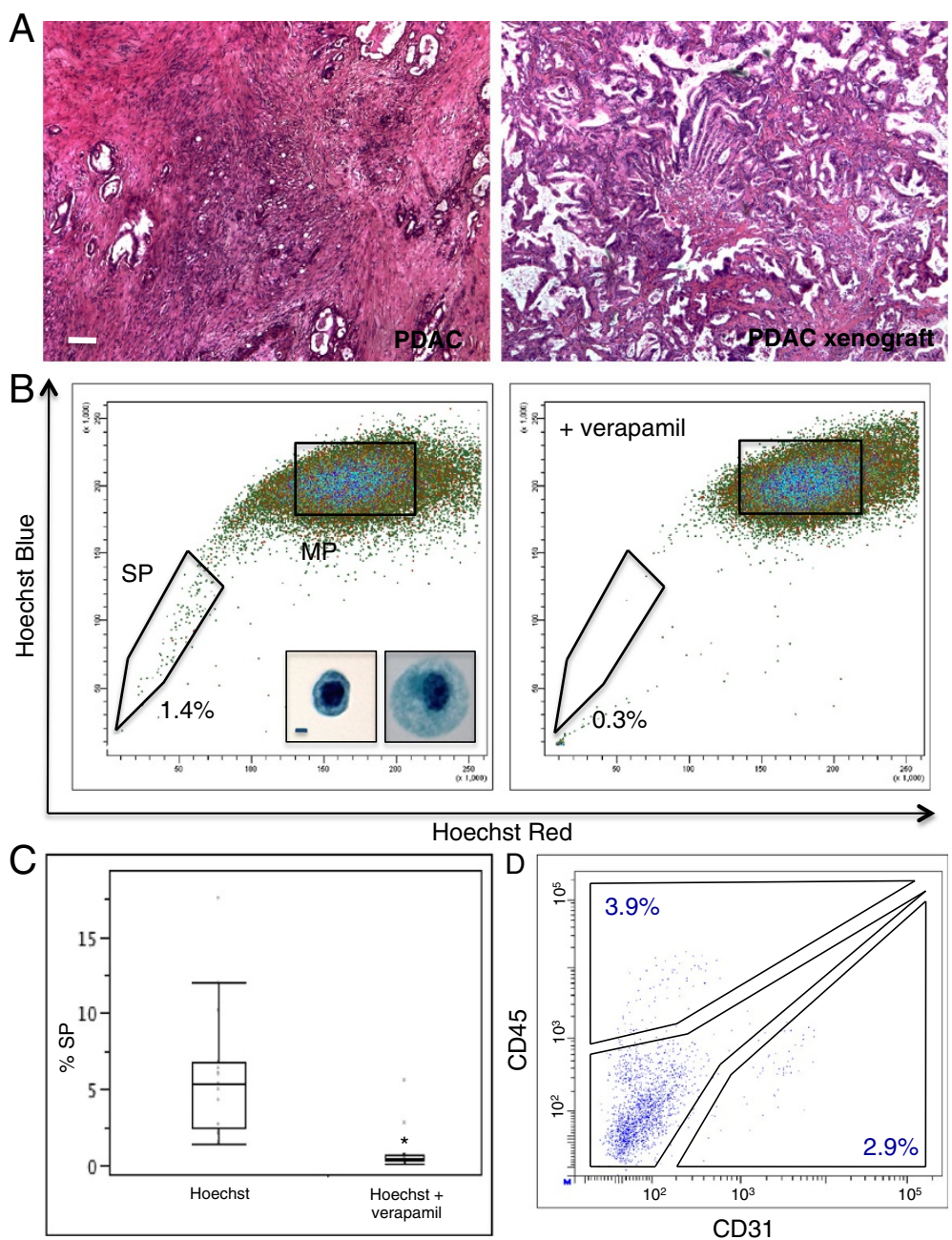

Figure 1 A SP is present in human PDAC. (A) Hematoxylin-Eosin staining of an original PDAC sample (left) and the corresponding xenograft tumour (right). A representative example is shown. Scale bar $=50 \mu \mathrm{m}$. (B) SP identification by FACS (left) and control of the SP phenotype with verapamil (right). A representative example with SP proportions is displayed. Inserts show a SP cell (left) and a MP cell (right) after Cyto Rich Red staining. Scale bar $=1 \mu \mathrm{m}$. (C) Boxplot of the SP proportion, with and without verapamil, of all PDAC xenografts analyzed $(n=17) .{ }^{*}, p<0.05$. (D) Analysis of CD45 and CD31 expression in the PDAC xenograft SP using FACS. A representative example is shown. Numbers represent the percentage of $\mathrm{CD}_{4} 5^{+}$cells and of $\mathrm{CD} 31^{+}$cells within the SP.

the SP phenotype (Figure 1B-C). The SP cells were smaller than the MP cells and had a larger nucleus/ cytoplasm ratio, indicative of a poorly differentiated or undifferentiated nature [3] (Figure 1B). No significant correlation was found between the SP size and patients' survival in this population of 17 PDAC (Table 1). Because it is known that the SP phenotype can co-purify endothelial and hematopoietic cells [20,23], we further analyzed the xenografts for $\mathrm{CD} 31^{+}$and $\mathrm{CD} 45^{+}$cells. Human epitopes were not detected (data not shown) but mouse $\mathrm{CD}_{4} 5^{+}$and $\mathrm{CD} 31^{+}$cells were found, indicating that host cells were attracted and incorporated into the growing human xenograft tumours. Yet, the proportion of $\mathrm{CD}_{4} 5^{+}$and $\mathrm{CD} 31^{+}$cells in the SP was low (median: $4.1 \%$ and $4.5 \%$, respectively; $\mathrm{n}=10$; Figure $1 \mathrm{D}$ ).
The SP is resistant to gemcitabine as assessed in vivo Seven different PDAC samples were grown in SCID mice (see Table 1). Treatment of mice with gemcitabine affected growth of 4 out of 7 xenograft tumours, resulting in an average volume reduction of $72 \%$ in comparison with an average tumour expansion in the vehicle-treated control group of $230 \%(\mathrm{p}=0.030$ ) (Figure $2 \mathrm{~A}-\mathrm{B})$. After treatment, tumours were excised and analyzed for SP. The tumour SP proportion was larger in mice treated with gemcitabine than in the corresponding controls (median $6.6 \%$ versus $2.7 \%$, respectively; $\mathrm{n}=7 ; \mathrm{p}=0.028$; Figure 2B-C). SP enrichment was even higher when only considering the tumours that responded to gemcitabine with tumour shrinkage (of at least 50\%) (median: 4.7\% versus $1.3 \%$, respectively; $\mathrm{n}=4$; Figure $2 \mathrm{~B}$ ). $\mathrm{CD} 45^{+}$and 

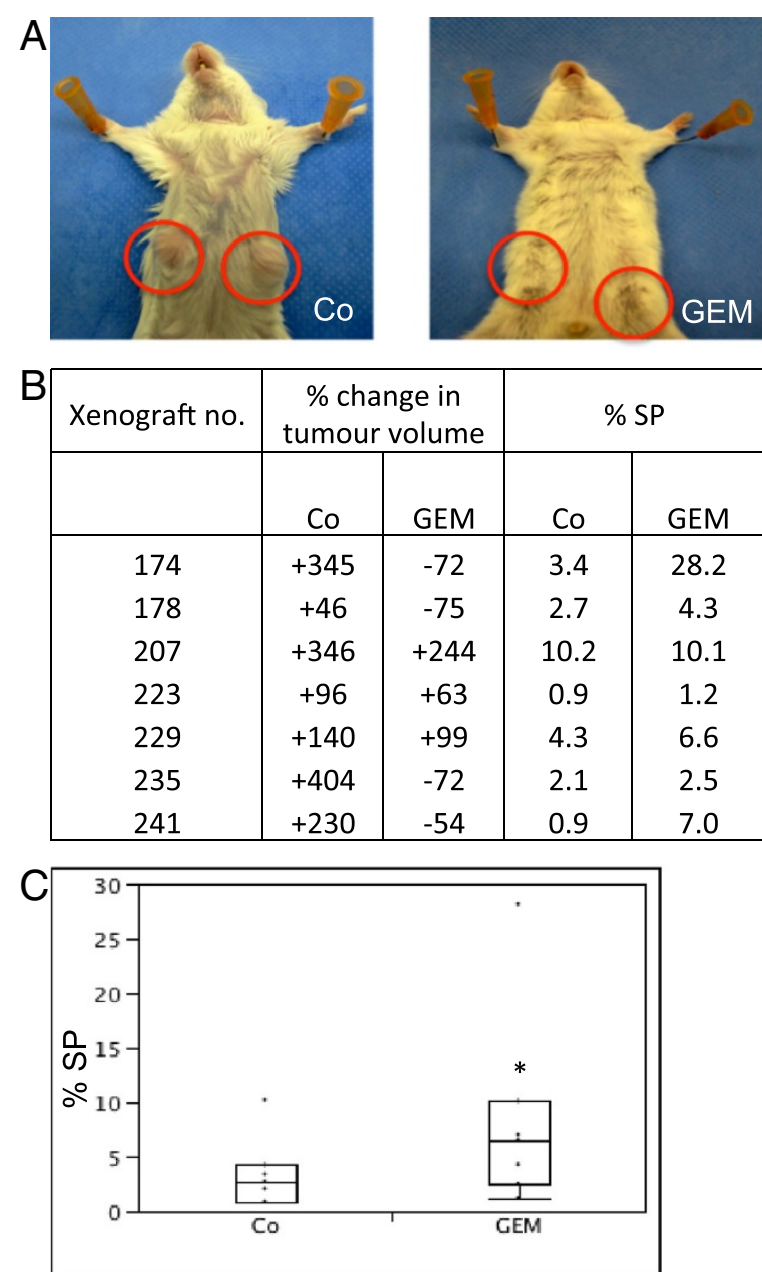

Figure $\mathbf{2}$ The SP in human PDAC is resistant to gemcitabine as analyzed in vivo. (A) SCID mice with corresponding PDAC xenograft tumours treated with vehicle (control or $\mathrm{Co}$, left) or gemcitabine (GEM, right). Tumours measured $276 \mathrm{~mm}^{3}$ and $266 \mathrm{~mm}^{3}$ in the control mouse and $144 \mathrm{~mm}^{3}$ and $123 \mathrm{~mm}^{3}$ in the GEM-treated mouse, respectively. (B) Overview of the data, indicating PDAC xenograft number, percent change in tumour volume after treatment with vehicle (Co) or GEM (response to GEM when $>50 \%$ reduction), and proportion of SP in the tumours after treatment. (C) Boxplot of SP proportion in the PDAC xenografts of the vehicle-treated control mice (CO) and the mice treated with $\operatorname{GEM}(n=7) .{ }^{*}, p<0.05$.

$\mathrm{CD}^{+} 1^{+}$cells did not rise in the SP in response to gemcitabine $(\mathrm{p}=0.21$ and $\mathrm{p}=0.66$, respectively) when compared to control mice.

\section{Whole-genome expression analysis of the PDAC xenograft SP reveals upregulation of genes related to therapy resistance}

To characterize the SP at the gene expression level, whole-genome expression analysis was performed on SP and MP cells sorted from 5 different xenograft tumours (see Table 1). Microarrays with human genome oligonucleotide probes were used, thereby excluding the detection of mouse transcripts (such as from the infiltrating $\mathrm{CD} 31^{+}$ and $\mathrm{CD}_{4} 5^{+} \mathrm{SP}$ cells). Comparison revealed that 145 probe sets, representing 121 genes, were differentially expressed between the SP and MP ( $<<0.001) ; 80$ genes were upregulated in the SP and 41 genes downregulated (complete list in Additional file 1: Table S1; extract of genes in Table 2, selected on the basis of relevance from the literature and from DAVID analysis as below).

Gene-clustering analysis of all differentially expressed genes $(\mathrm{p}<0.001)$ by DAVID showed 3 functionally related groups of genes enriched in the SP: one group of transcription factors, one of adhesion molecules and one of homeobox genes (Table 3). KEGG pathway analysis revealed 3 significantly upregulated pathways in the $\mathrm{SP}$ versus the MP, including 'cancer' and 'adherens junctions' (Table 3). Two KEGG pathways were significantly downregulated in the SP, including 'cell adhesion molecules'. Visualization of the interaction network of SP-upregulated genes by STRING analysis (Figure 3) reveals that genes involved in chemoresistance [ETS1, KIT ligand (KITLG) or stem cell factor (SCF), SNAI2], regulation of apoptosis (FASLG, GRB10, BCL2L11, ETS1, SNAI2), epithelial-mesenchymal transition (EMT) (SNAI2, LEF1) and tumourigenesis (oncogenes like FGF7, GATA1, KITLG, ETS1) occupy a central position. Moreover, multidrug transporters, linked to chemoresistance and some considered responsible for the SP phenotype, also show a clear tendency of upregulation in the SP (ABCG2, 3.63 fold, $\mathrm{p}=0.006 ; A B C A 9$, 3.66-fold, $\mathrm{p}=0.003$ ).

\section{The SP is enriched in sphere-forming cells}

Tumourigenic (CSC) activity was analyzed in vitro using the sphere-forming assay [22]. SP and MP from xenograft tumours were first depleted from the (murine) endothelial and immune cells by FACS and then seeded in defined culture conditions (see Methods). Viability of the sorted SP and MP cells was identical (data not shown). The $\mathrm{CD} 45^{\circ} / \mathrm{CD} 31^{-} \mathrm{SP}$ generated spheres in all experiments ( $\mathrm{n}=6$; median number of spheres: 16; range: 10-35; Figure 4). In contrast, the CD45/CD31- MP did not consistently generate spheres (not in 2 out of the 6 experiments) and the spheres obtained were lower in number (range: $0-15$; median: $8 ; \mathrm{p}=0.016$ versus $\mathrm{SP}$ ), less wellformed, and smaller in size (Figure 4). These findings indicate that SP cells have a higher sphere-forming capacity than MP cells. It was not possible to assess the propagation (self-renewal) capacity of the sphere-forming cells because the number of spheres obtained was too low and the dispersion did not yield enough (viable) cells.

\section{Discussion}

To date, SP analysis in pancreatic cancer has been limited to cultured cell lines $[6-8,24,25]$. In the present 
Table 2 Selection of genes differentially expressed in the PDAC xenograft SP versus MP

\begin{tabular}{|c|c|c|c|c|}
\hline Gene Symbol & Genbank Accession no. & Gene Name & Fold SP/MP & p-value \\
\hline ZAP70 & NM_001079 & zeta-chain (TCR) associated protein kinase $70 \mathrm{kDa}$ & 24.25 & 0.00002 \\
\hline PRKCQ & NM_006257 & protein kinase $C$, theta & 21.56 & 0.00008 \\
\hline FASLG & NM_000639 & Fas ligand (TNF superfamily, member 6) & 19.56 & 0.00003 \\
\hline LEF1 & NM_000639 & lymphoid enhancer-binding factor 1 & 16.45 & 0.00009 \\
\hline PACSIN1 & NM_020804 & protein kinase $C$ and casein kinase substrate in neurons 1 & 12.47 & 0.00015 \\
\hline MEOX2 & NM_005924 & mesenchyme homeobox 2 & 8.22 & 0.00060 \\
\hline STAT4 & NM_003151 & signal transducer and activator of transcription 4 & 7.46 & 0.00050 \\
\hline EMX2 & NM_003151 & empty spiracles homeobox 2 & 6.87 & 0.00006 \\
\hline SOX11 & NM_003108 & SRY (sex determining region Y)-box 11 & 6.19 & 0.00029 \\
\hline FGF7 & NM_002009 & fibroblast growth factor 7 (keratinocyte growth factor) & 5.74 & 0.00072 \\
\hline FOXG1B & NM_005249 & forkhead box G1B & 5.62 & 0.00019 \\
\hline PKNOX2 & NM_022062 & PBX/knotted 1 homeobox 2 & 5.58 & 0.00017 \\
\hline ITGB3 & NM_000212 & integrin, beta 3 (platelet glycoprotein IIla, antigen CD61) & 5.35 & 0.00062 \\
\hline KLF12 & NM_007249 & Kruppel-like factor 12 & 5.03 & 0.00048 \\
\hline SNAI2 & NM_003068 & snail homolog 2 (Drosophila) & 4.99 & 0.00079 \\
\hline TIE1 & NM_005424 & tyrosine kinase with immunoglobulin-like and EGF-like domains 1 & 4.76 & 0.00019 \\
\hline $\operatorname{IRX} 2$ & AY335940 & iroquois homeobox protein 2 & 4.53 & 0.00010 \\
\hline EFNB2 & NM_004093 & ephrin-B2 & 4.23 & 0.00016 \\
\hline ETS1 & NM_005238 & v-ets erythroblastosis virus E26 oncogene homolog 1 (avian) & 3.95 & 0.00095 \\
\hline BCL2L11 & NM_138621 & BCL2-like 11 & 3.68 & 0.00019 \\
\hline GRB10 & NM_001001555 & growth factor receptor-bound protein 10 & 3.68 & 0.00034 \\
\hline NFIB & NM_005596 & nuclear factor I/B & 3.46 & 0.00010 \\
\hline INHBB & NM_002193 & inhibin, beta B (activin AB beta polypeptide) & 3.32 & 0.00059 \\
\hline TCF7L1 & NM_031283 & transcription factor 7-like 1 (T-cell specific, HMG-box) & 3.14 & 0.00073 \\
\hline EPC2 & NM_053001 & enhancer of polycomb homolog 2 (Drosophila) & 2.95 & 0.00090 \\
\hline GATA1 & NM_002049 & GATA binding protein 1 (globin transcription factor 1) & 2.57 & 0.00082 \\
\hline KITLG & NM_000899 & KIT ligand & 2.50 & 0.00052 \\
\hline ERRFI1 & NM_018948 & ERBB receptor feedback inhibitor 1 & 2.45 & 0.00074 \\
\hline CADM1 & NM_014333 & cell adhesion molecule 1 & -22.84 & 0.00077 \\
\hline TREM2 & NM_018965 & triggering receptor expressed on myeloid cells 2 & -9.90 & 0.00017 \\
\hline ALOX5AP & NM_001629 & arachidonate 5-lipoxygenase-activating protein & -9.32 & 0.00017 \\
\hline PLA2G7 & NM_005084 & phospholipase A2, group VII (platelet-activating factor acetylhydrolase, plasma) & -7.66 & 0.00007 \\
\hline CD74 & NM_004355 & CD74 molecule, major histocompatibility complex, class II invariant chain & -7.28 & 0.00006 \\
\hline MADCAM1 & NM_130760 & mucosal vascular addressin cell adhesion molecule 1 & -6.53 & 0.00004 \\
\hline CD14 & NM_130760 & CD14 molecule & -5.94 & 0.00038 \\
\hline CTSA & NM_000308 & cathepsin A & -3.29 & 0.00068 \\
\hline CTSC & NM_001814 & cathepsin C & -2.97 & 0.00085 \\
\hline
\end{tabular}

study, we demonstrate for the first time the presence of a $\mathrm{SP}$ in human PDAC samples using first-generation xenografts, histologically resemblant to the original tumour samples except for the stromal component. We showed, using a xenograft in vivo model, that the SP is more resistant to gemcitabine than the other tumour cells, and therefore may represent a potential therapeutic target.
The response rate of the xenograft tumours to gemcitabine ( 4 out of 7 ) was higher than the known response rate in human patients (lower than 20\%), which may be due to the reduced stromal component in the xenografts, thus improving drug delivery to the tumour cells (see $[3,4]$ ).

Whole-genome expression profiling of the SP demonstrated the expression of genes involved in cancer 
Table 3 Gene-function analysis of all differentially expressed genes between PDAC xenograft SP and MP using DAVID A. ENRICHED GENE CLUSTERS Gene group 1: Transcription Factors Enrichment Score: 1.78

2

3

4

5

6

7

8

9

10

Gene group 2: Adhesion Molecules Enrichment Score: 1.71

$\begin{array}{ll}1 & \text { HLA-DPB1 } \\ 2 & \text { MADCAM1 } \\ 3 & \text { TREM2 } \\ 4 & \text { EFNB2 } \\ 5 & \text { IGSF6 } \\ 6 & \text { LRFN5 } \\ 7 & \\ 8 & \text { DSCAM } \\ 9 & \text { JAM2 } \\ & \text { MDGA1 }\end{array}$

Gene group 3: Homeobox Genes Enrichment Score: 1.51

$\begin{array}{ll}1 & \text { PKNOX2 } \\ 2 & \text { MEOX2 } \\ 3 & \text { SPIC } \\ 4 & \text { IRX2 } \\ 5 & \text { EMX2 }\end{array}$

\section{B. KEGG PATHWAYS}

Upregulated in SP

Arrhythmogenic rightventricular

4 cardiomyopathy

Pathways in cancer

6

Adherens junction
Number of genes

TCF7L1

SNAI2

CNOT4

ZBTB10

KLF12

ZFPM2

STAT4

OSR2

IKZF2

PHF6

ZFHX4

SOX11

CITED4

ARNTL

NFIB

GATA1

ETS

LEF1

HLA-DPB1

REM2

SF6

PKNOX2

MEOX2

IRX2

3 transcription factor 7-like 1 (T-cell specific, HMG-box)

snail homolog 2 (Drosophila)

CCR4-NOT transcription complex, subunit 4

zinc finger and BTB domain containing 10

Kruppel-like factor 12

zinc finger protein, multitype 2

signal transducer and activator of transcription 4

odd-skipped related 2 (Drosophila)

IKAROS family zinc finger 2 (Helios)

PHD finger protein 6

zinc finger homeobox 4

SRY (sex determining region Y)-box 11

Cbp/p300-interacting transactivator, with Glu/ Asp-rich carboxy-terminal domain, 4

aryl hydrocarbon receptor nuclear translocator-like nuclear factor I/B

GATA binding protein 1 (globin transcription factor 1) v-ets erythroblastosis virus E26 oncogene homolog 1 (avian)

lymphoid enhancer-binding factor 1

major histocompatibility complex, class II, DP beta 1 mucosal vascular addressin cell adhesion molecule 1 triggering receptor expressed on myeloid cells 2 ephrin-B2 immunoglobulin superfamily, member 6 leucine rich repeat and fibronectin type III domain containing 5

Down syndrome cell adhesion molecule junctional adhesion molecule 2

MAM domain containing glycosylphosphatidylinositol anchor 1

PBX/knotted 1 homeobox 2

mesenchyme homeobox 2

Spi-C transcription factor (Spi-1/PU.1 related)

iroquois homeobox 2

empty spiracles homeobox 2

genes

GJA1, ITGB3, LEF1, TCFL1

TCF7L1, KITL, FASL, LEF1, FGF7, ETS1

LEF1, SNAI2, TCF7L1 
Table 3 Gene-function analysis of all differentially expressed genes between PDAC xenograft SP and MP using DAVID (Continued)

\begin{tabular}{lll}
\hline Downregulated in SP & Number of genes & genes \\
Lysosome & 3 & CTSA, CTSC, SLC11A1 \\
Cell adhesion molecules (CAMs) & 3 & CADM1, HLA-DPB1, MADCAM1 \\
\hline
\end{tabular}

pathways, particularly chemoresistance and EMT. In many cancers, the proto-oncogene ETS1 plays a role in chemoresistance and invasion [26]. In PDAC cell lines, ETS1 expression has been linked to gemcitabine resistance and to invasiveness by induction of matrix metalloproteinase (MMP) 2 [27]. We also found upregulation of $M M P 2$ in the PDAC SP, although at the border of statistical significance (3.12 fold; $\mathrm{p}=0.003)$. Equally, KITLG $(S C F)$ increases invasive capacity in PDAC cell lines, as well as cell proliferation $[28,29]$. The zinc finger transcription factor SNAI2 (SLUG) is involved in chemo- and radioresistance (e.g. through anti-apoptotic mechanisms) [30].
Moreover, SNAI2 is a core regulator of EMT, a key process in cancer pathogenesis and tumour progression by which epithelial cells acquire a mesenchymal phenotype with invasive and migratory properties. EMT is considered to play an important role in tumour resistance and metastasis. The SP of the pancreatic cell line PANC1 has been shown to possess superior potential for EMT when compared to the MP [24]. Recently, SNAI2 was shown to promote EMT, invasion and metastasis in a pancreatic cancer cell line [31,32]. EMT involves the downregulation of cell adhesion molecules like E-cadherin. In the present study, E-cadherin was significantly downregulated

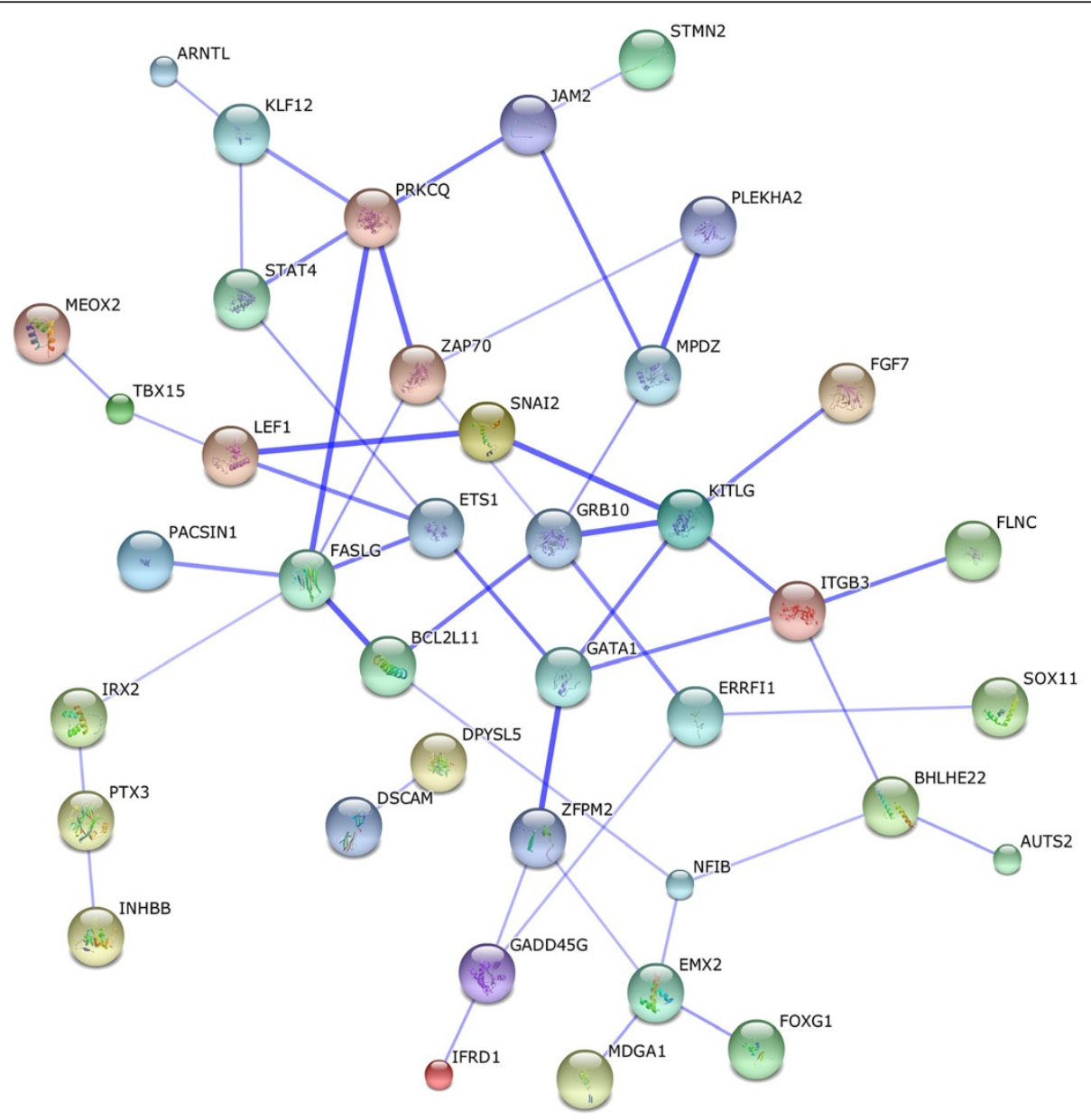

Figure 3 Interaction network of genes upregulated in the human PDAC SP. STRING analysis of genes upregulated in the human PDAC xenograft SP versus the MP (displayed as 'evidence view', i.e. only connected nodes are shown). 

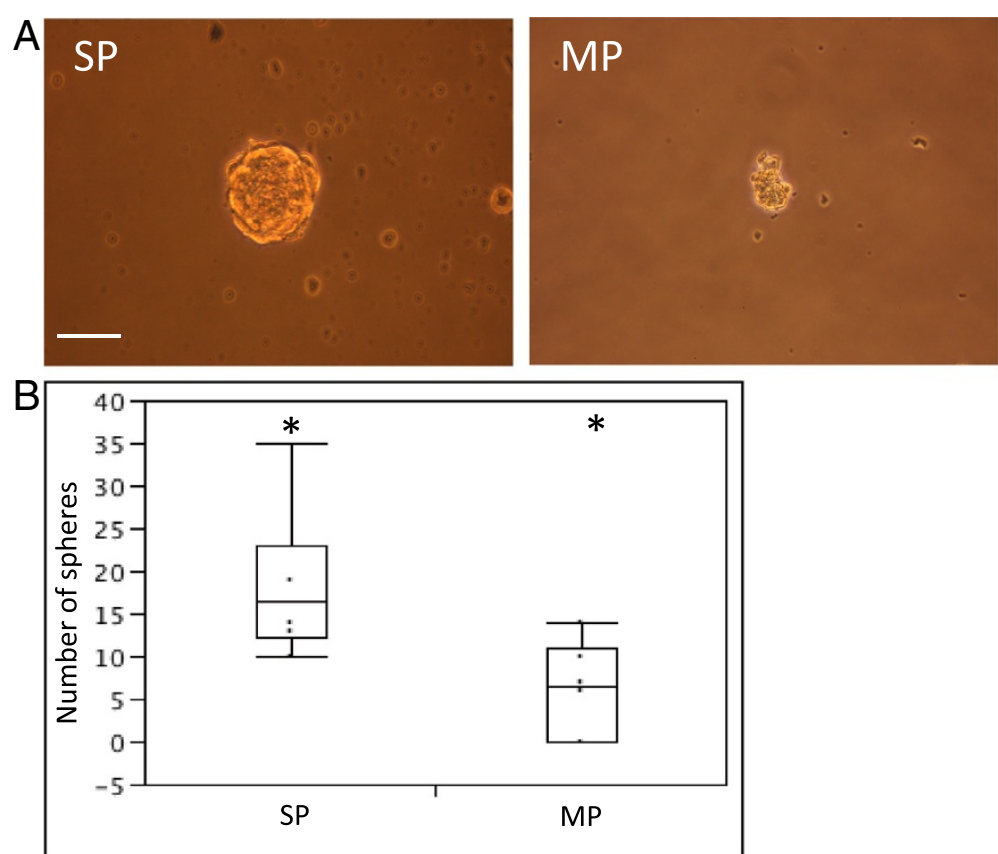

Figure 4 The human PDAC SP displays higher sphere-forming capacity than the MP. (A) Spheres grown from CD45/CD31 SP cells (left) and $\mathrm{CD}_{45} / \mathrm{CD} 31^{-} \mathrm{MP}$ cells (right) sorted from human PDAC xenografts. Representative examples are shown. Scale bar $=50 \mu \mathrm{m}$. (B) Boxplot of the number of spheres obtained $(n=6) .{ }^{*}, p<0.05$.

in 3 of the 5 PDAC samples analyzed (data not shown). SNAI2 can act as a transcriptional repressor of E-cadherin. However, in pancreatic cancer cell lines, SNAI2 was found only a weak suppressor of E-cadherin [32], whereas in human PDAC, no significant correlation could be observed between SNAI2 and E-cadherin [32,33]. LEF1 (lymphoid enhancer factor 1), a nuclear transducer of the Wnt signaling pathway, also plays a key role in EMT. In addition, LEF1 expression in human pancreatic cancer correlates with advanced tumour stages [34]. TCF7L1 is known to form a complex with LEF1 to achieve DNAbinding ability. Finally, other signaling proteins of the Wnt pathway (Wnt7b, DVL1, FZD1, FZD4, FZD5) as well as components of the TGF $\beta / B M P$ pathway (TGF $\beta 1$, $B M P 1, B M P P 2 R a, S M U R F 2)$ were upregulated in the SP versus $\mathrm{MP}$, although not reaching statistical significance (0.001 < p $<0.05$; data not shown).

Protection against apoptosis represents a further mechanism of therapy resistance. In our current study, BCL2L11 and FASLG are upregulated in the SP. In studies with pancreatic cancer cell lines, BCL2L11 has been correlated with apoptotic resistance as well as with metastatic potential [35,36]. FASLG may regulate immune evasion of tumour cells by inducing apoptosis in cytotoxic T lymphocytes. FASLG has been reported to play a role in the aggressiveness of PDAC, potentially through this immune escape mechanism [37].
Finally, the multidrug transporter $A B C G 2$ is highly expressed in the SP, suggesting a role in chemoresistance and at the same time supporting the SP phenotype which is, at least partially, linked to activity of this pump. In pancreatic cancer cell lines, $A B C G 2$ was also found to be upregulated in the SP $[6,8]$ and associated with chemotherapy resistance [38]. In addition, $A B C A 9$, another membrane transporter linked to chemoresistance in some cancers like malignant melanoma [39], was found upregulated in the PDAC SP in our study.

In multiple types of cancer, the SP displays properties reminiscent of CSC. In the present study, we show that the SP of PDAC is enriched in cells that generate spheres. Although sphere formation is regarded as an in-vitro assay for CSC (tumourigenic) activity (see e.g. [22]), it should be noted that this link is not always present as reported, for instance, in high-grade glioma [40]. In order to demonstrate tumourigenic (CSC) activity of the PDAC SP, further study is needed that analyzes in-vivo tumour growth after implantation of the (purified) population in immunodeficient mice. Given the essential interactions of cancer cells with their microenvironment for tumour development, transplantation within the pancreas would be most appropriate but technically highly demanding. Yet, some expression characteristics may already suggest a CSC(-like) phenotype including expression of genes associated with chemoresistance (see above), with the 
Wnt pathway and with EMT. Indeed, the latter process has recently been uncovered as a key promoter of the generation and activity of CSC [41]. Moreover, KITLG (SCF) has been linked to CSC in prostate [42] and lung cancer [43]. In the present study, expression of the previously proposed CSC membrane markers in pancreatic cancer (i.e. CD $24^{+} \mathrm{CD} 44^{+} \mathrm{ESA}^{+}[16,44]$ and $\mathrm{CD} 133^{+}$[17]) was not found upregulated in the PDAC xenograft SP, neither in microarray analysis, nor in flow-cytometric examination $\left(1.0 \% \mathrm{CD}_{133^{+}}\right.$cells in the SP and $0.7 \%$ in the MP; $\mathrm{n}=2$ ) (data not shown). Noteworthy, expression of the two previously defined sets of markers $\left(\mathrm{CD} 24^{+} \mathrm{CD} 44^{+} \mathrm{ESA}^{+}\right.$ and $\mathrm{CD} 133^{+}$) did also not completely, or only minimally, overlap (10-40\%) [16]. Thus, CSC in pancreatic cancer, and the link with the SP, need further investigation.

\section{Conclusions}

Our study revealed the presence of a SP in human PDAC, displaying chemoresistance and CSC-associated activity, as well as expression of genes involved in both processes. SP cells thus may represent interesting targets for new and more efficient therapeutic strategies. Chemoresistance, anti-apoptosis and EMT genes identified may guide us to potential molecular targets.

\section{Additional file}

Additional file 1: Table S1. Complete list of genes differentially expressed in the PDAC SP versus MP $(p<0.001)$.

\section{Abbreviations}

PDAC: Pancreatic ductal adenocarcinoma; SP: Side population; MP: Main population; FACS: Fluorescence-activated cell sorter; CSC: Cancer stem cell(s); s.c.: Subcutaneously; SCID: Severe combined immunodeficiency; DAVID: Database for annotation, Visualization and Integrated Discovery; KEGG: Kyoto Encyclopedia of Genes and Genomes; STRING: Search Tool for the Retrieval of Interacting Genes/Proteins; EMT: Epithelial-mesenchymal transition.

\section{Competing interests}

The authors declare that they have no competing interests

\section{Authors' contributions}

AVDB designed and performed the study, analyzed the data and wrote the manuscript. LG trained AVDB in the techniques used and helped in data collection. BT participated in study design and analysis and in writing. HV participated in the design and analysis of the study and wrote the manuscript. All authors read and approved the final manuscript.

\section{Acknowledgements}

AVDB and LG acknowledge support by PhD Fellow grants from the Fund for Scientific Research - Flanders (FWO-Vlaanderen) and BT acknowledges support by a research grant of the FWO.

The authors are very grateful to Vik Van Duppen (Hematology, KU Leuven) for his help with FACS analysis and sorting, and to the VIB-MAF (KU Leuven) for the microarray analysis.

\section{Author details}

'Department of Abdominal Surgery, University Hospitals Leuven, Herestraat 49, B-3000, Leuven, Belgium. ${ }^{2}$ Laboratory of Tissue Plasticity, Research Unit of Embryo and Stem Cells, Department of Development \& Regeneration, University of Leuven (KU Leuven), Leuven, Belgium.
Received: 10 March 2012 Accepted: 30 July 2012

Published: 15 August 2012

\section{References}

1. Jemal A, Siegel R, Ward E, Hao Y, XU J, Murray T, Thun MJ: Cancer statistics, 2008. CA Cancer J Clin 2008, 58(2):71-96.

2. Van den Broeck A, Sergeant G, Ectors N, Van Steenbergen W, Aerts R, Topal B: Patterns of recurrence after curative resection of pancreatic ductal adenocarcinoma. Eur J Surg Oncol 2009, 35(6):600-604.

3. Olive KP, Jacobetz MA, Davidson CJ, Gopinathan A, McIntyre D, Honess D, Madhu B, Goldgraben MA, Caldwell ME, Allard D, Frese KK, Denicola G, Feig C, Combs C, Winter SP, Ireland-Zecchini H, Reichelt S, Howat WJ, Chang A, Dhara M, Wang L, Rückert F, Grützmann R, Pilarsky C, Izeradjene K, Hingorani SR, Huang P, Davies SE, Plunkett W, Egorin M, et al: Inhibition of Hedgehog signaling enhances delivery of chemotherapy in a mouse model of pancreatic cancer. Science 2009, 324(5933):1457-1461.

4. Olson P, Hanahan D: Cancer. Breaching the cancer fortress. Science 2009, 324(5933):1400-1401.

5. Goodell MA: Stem cell identification and sorting using the Hoechst 33342 side population (SP). Curr Protoc Cytom 2005, suppl 9:18.1-18.11.

6. Yao J, Cai HH, Wei JS, An Y, Ji ZL, Lu ZP, Wu JL, Chen P, Jiang KR, Dai CC, Qian ZY, Xu ZK, Miao Y: Side population in the pancreatic cancer cell lines SW1990 and CFPAC-1 is enriched with cancer stem-like cells. Oncol Rep 2010, 23(5):1375-1382.

7. Zhang SN, Huang FT, Huang YJ, Zhong W, Yu Z: Characterization of a cancer stem cell-like side population derived from human pancreatic adenocarcinoma cells. Tumori 2010, 96(6):985-992.

8. Zhou J, Wang CY, Liu T, Wu B, Zhou F, Xiong JX, Wu HS, Tao J, Zhao G, Yang M, Gou SM: Persistence of side population cells with high drug efflux capacity in pancreatic cancer. World J Gastroenterol 2008, 14(6):925-930

9. Haraguchi N, Utsunomiya T, Inoue H, Tanaka F, Mimori K, Barnard GF, Mori M: Characterization of a side population of cancer cells from human gastrointestinal system. Stem Cells 2006, 24(3):506-513.

10. Wu C, Alman BA: Side population cells in human cancers. Cancer Lett 2008, 268(1):1-9.

11. Clarke MF, Dick JE, Dirks PB, Eaves CJ, Jamieson CH, Jones DL, Visvader J, Weissman IL, Wahl GM: Cancer stem cells-perspectives on current status and future directions: AACR Workshop on cancer stem cells. Cancer Res 2006, 66(19):9339-9344.

12. Sergeant G, Vankelecom $H$, Gremeaux L, Topal B: Role of cancer stem cells in pancreatic ductal adenocarcinoma. Nat Rev Clin Oncol 2009, 6(10):580-586

13. Boman BM, Wicha MS: Cancer stem cells: a step toward the cure. J Clin Oncol 2008, 26(17):2795-2799.

14. Eaves CJ: Cancer stem cells: Here, there, everywhere? Nature 2008, 456(7222):581-582.

15. Eppert K, Takenaka K, Lechman ER, Waldron L, Nilsson B, van Galen P, Metzeler KH, Poeppl A, Ling V, Beyene J, Canty AJ, Danska JS, Bohlander SK, Buske C, Minden MD, Golub TR, Jurisica I, Ebert BL, Dick JE: Stem cell gene expression programs influence clinical outcome in human leukemia. Nat Med 2011, 17(9):1086-1093.

16. Li C, Heidt DG, Dalerba P, Burant CF, Zhang L, Adsay V, Wicha M, Clarke MF, Simeone DM: Identification of pancreatic cancer stem cells. Cancer Res 2007, 67(3):1030-1037.

17. Hermann PC, Huber SL, Herrler T, Aicher A, Ellwart JW, Guba M, Bruns CJ, Heeschen C: Distinct populations of cancer stem cells determine tumor growth and metastatic activity in human pancreatic cancer. Cell Stem Cell 2007, 1(3):313-323.

18. Tomayko MM, Reynolds CP: Determination of subcutaneous tumor size in athymic (nude) mice. Cancer Chemother Pharmacol 1989, 24(3):148-154.

19. Baugh $L R$, Hill $A A$, Brown EL, Hunter CP: Quantitative analysis of mRNA amplification by in vitro transcription. Nucleic Acids Res 2001, 29(5):E29.

20. Chen J, Gremeaux L, Fu Q, Liekens D, Van Laere S, Vankelecom H: Pituitary progenitor cells tracked down by side population dissection. Stem Cells 2009, 27(5):1182-1195.

21. Smyth GK: Linear models and empirical bayes methods for assessing differential expression in microarray experiments. Stat Appl Genet Mol Biol 2004, 3(1):1544-6115. 
22. Gou S, Liu T, Wang C, Yin T, Li K, Yang M, Zhou J: Establishment of clonal colony-forming assay for propagation of pancreatic cancer cells with stem cell properties. Pancreas 2007, 34(4):429-435

23. Bleau AM, Hambardzumyan D, Ozawa T, Fomchenko El, Huse JT, Brennan CW, Holland EC: PTEN/PI3K/Akt pathway regulates the side population phenotype and ABCG2 activity in glioma tumor stem-like cells. Cell Stem Cell 2009, 4(3):226-235.

24. Kabashima A, Higuchi $H$, Takaishi H, Matsuzaki Y, Suzuki S, Izumiya M, lizuka H, Sakai G, Hozawa S, Azuma T, Hibi T: Side population of pancreatic cancer cells predominates in TGF-beta-mediated epithelial to mesenchymal transition and invasion. Int J Cancer 2009, 124(12):2771-2779.

25. Wang YH, Li F, Luo B, Wang XH, Sun HC, Liu S, Cui YQ, Xu XX: A side population of cells from a human pancreatic carcinoma cell line harbors cancer stem cell characteristics. Neoplasma 2009, 56(5):371-378.

26. Dittmer J: The biology of the Ets1 proto-oncogene. Mol Cancer 2003, 2:29

27. Khanna A, Mahalingam K, Chakrabarti D, Periyasamy G: Ets-1 expression and gemcitabine chemoresistance in pancreatic cancer cells. Cell Mol Biol Lett 2011, 16(1):101-113.

28. Zhang M, Ma Q, Hu H, Zhang D, Li J, Ma G, Bhat K, Wu E: Stem cell factor/c-kit signaling enhances invasion of pancreatic cancer cells via HIF-1alpha under normoxic condition. Cancer Lett 2011, 303(2):108-117.

29. Yasuda A, Sawai H, Takahashi H, Ochi N, Matsuo Y, Funahashi H, Sato M, Okada Y, Takeyama H, Manabe T: The stem cell factor/c-kit receptor pathway enhances proliferation and invasion of pancreatic cancer cells. Mol Cancer 2006, 5:46.

30. Cobaleda C, Perez-Caro M, Vicente-Duenas C, Sanchez-Garcia I: Function of the zinc-finger transcription factor SNAI2 in cancer and development. Annu Rev Genet 2007, 41:41-61.

31. Nishioka R, Itoh S, Gui T, Gai Z, Oikawa K, Kawai M, Tani M, Yamaue H, Muragaki Y: SNAIL induces epithelial-to-mesenchymal transition in a human pancreatic cancer cell line (BxPC3) and promotes distant metastasis and invasiveness in vivo. Exp Mol Pathol 2010, 89(2):149-157.

32. Hotz B, Arndt M, Dullat S, Bhargava S, Buhr HJ, Hotz HG: Epithelial to mesenchymal transition: expression of the regulators snail, slug, and twist in pancreatic cancer. Clin Cancer Res 2007, 13(16):4769-4776.

33. Zhang K, Chen D, Jiao X, Zhang S, Liu X, Cao J, Wu L, Wang D: Slug enhances invasion ability of pancreatic cancer cells through upregulation of matrix metalloproteinase- 9 and actin cytoskeleton remodeling. Lab Invest 2011, 91(3):426-438.

34. Jesse $S$, Koenig A, Ellenrieder V, Menke A: Lef-1 isoforms regulate different target genes and reduce cellular adhesion. Int J Cancer 2010, 126(5):1109-1120.

35. Ruckert F, Samm N, Lehner AK, Saeger HD, Grutzmann R, Pilarsky C: Simultaneous gene silencing of $\mathrm{BCl}-2, \mathrm{XIAP}$ and Survivin re-sensitizes pancreatic cancer cells towards apoptosis. BMC Cancer 2010, 10:379.

36. Bold RJ, Virudachalam S, McConkey DJ: BCL2 expression correlates with metastatic potential in pancreatic cancer cell lines. Cancer 2001 92(5):1122-1129.

37. Ohta T, Elnemr A, Kitagawa H, Kayahara M, Takamura H, Fujimura T, Nishimura G, Shimizu K, Yi SQ, Miwa K: Fas ligand expression in human pancreatic cancer. Oncol Rep 2004, 12(4):749-754.

38. Chen M, Xue X, Wang F, An Y, Tang D, Xu Y, Wang H, Yuan Z, Gao W, Wei J, Zhang J, Miao Y: Expression and promoter methylation analysis of ATPbinding cassette. Oncol Rep 2012, 27(1):265-269.

39. Chen KG, Valencia JC, Gillet JP, Hearing VJ, Gottesman MM: Involvement of $A B C$ transporters in melanogenesis and the development of multidrug resistance of melanoma. Pigment Cell Melanoma Res 2009, 22(6):740-749.

40. Barrett LE, Granot Z, Coker C, lavarone A, Hambardzumyan D, Holland EC, Nam HS, Benezra R: Self-renewal does not predict tumor growth potential in mouse models of high-grade glioma. Cancer Cell 2012, 21(1):11-24.

41. Mani SA, Guo W, Liao MJ, Eaton EN, Ayyanan A, Zhou AY, Brooks M, Reinhard F, Zhang CC, Shipitsin M, Campbell LL, Polyak K, Brisken C, Yang J, Weinberg RA: The epithelial-mesenchymal transition generates cells with properties of stem cells. Cell 2008, 133(4):704-715.

42. Ma Y, Liang D, Liu J, Axcrona K, Kvalheim G, Giercksky KE, Nesland JM, Suo Z: Synergistic effect of SCF and G-CSF on stem-like properties in prostate cancer cell lines. Tumour Bio/ 2012, 33(4):967-978.

43. Levina V, Marrangoni A, Wang T, Parikh S, Su Y, Herberman R, Lokshin A, Gorelik E: Elimination of human lung cancer stem cells through targeting of the stem cell factor-c-kit autocrine signaling loop. Cancer Res 2010, 70(1):338-346.

44. Simeone DM: Pancreatic cancer stem cells: implications for the treatment of pancreatic cancer. Clin Cancer Res 2008, 14(18):5646-5648.

doi:10.1186/1471-2407-12-354

Cite this article as: Van den broeck et al:: Human pancreatic adenocarcinoma contains a side population resistant to gemcitabine. BMC Cancer 2012 12:354.

\section{Submit your next manuscript to BioMed Central and take full advantage of:}

- Convenient online submission

- Thorough peer review

- No space constraints or color figure charges

- Immediate publication on acceptance

- Inclusion in PubMed, CAS, Scopus and Google Scholar

- Research which is freely available for redistribution

Submit your manuscript at www.biomedcentral.com/submit
C) BioMed Central 